\title{
A New Angle on Angle Surgery
}

\author{
Rhys Davies, Mei-Ling Cheng and Andrew J Tatham \\ Princess Alexandra Eye Pavilion and Department of Ophthalmology, University of Edinburgh, Edinburgh, Scotland, UK
}

DOl: https://doi.org/10.17925/EOR.2017.11.01.40

\begin{abstract}
$\mathrm{G}$ laucoma surgery has long relied on ab externo techniques such as augmented trabeculectomy and tube-shunt procedures. While these have excellent potential to lower intraocular pressure (IOP), they are associated with risks, including hypotony, and are therefore conventionally reserved for those with advanced glaucoma or at high risk of visual loss. Traditional filtering surgery attempts to bypass the eye's physiological outflow pathway, however new minimally invasive glaucoma surgery (MIGS) procedures have recently been introduced that focus on the angle structures as a target for improving aqueous outflow. There are a growing number of $a b$ interno MIGS procedures that utilise the natural drainage pathways of the eye in the management of glaucoma. This article examines the progress so far and describes how the angle has become a viable target for glaucoma surgery.
\end{abstract}

\section{Keywords}

Minimally invasive glaucoma surgery (MIGS), ab interno, glaucoma, angle surgery

Disclosure: Rhys Davies, Mei-Ling Cheng and Andrew J Tatham have nothing to declare in relation to this article. This study involves a review of the literature and did not involve any studies with human or animal subjects performed by any of the authors. No funding was received for the publication of this article.

Authorship: All named authors meet the International Committee of Medical Journal Editors (ICMJE) criteria

for authorship of this manuscript, take responsibility

for the integrity of the work as a whole, and have given final approval to the version to be published.

open Access: This article is published under the Creative Commons Attribution Noncommercial License which permits any non-commercial use, distribution, adaptation and reproduction provided the original author(s) and source are given appropriate credit.

Received: 28 April 2017

Accepted: 15 June 2017

Citation: European Ophthalmic Review, 2017;11(1):40-3

Corresponding Author: Andrew J Tatham, Princess Alexandra Eye Pavilion and Department of

Ophthalmology, University of Edinburgh, Chalmers Street, Edinburgh, Scotland, EH3 9HA, UK. E: atatham@nhs.net
The major risk factor for primary open angle glaucoma (POAG) is raised intraocular pressure (IOP), which is a result of increased resistance to aqueous humour outflow, thought to occur predominantly at the level of the trabecular meshwork (TM) and Schlemm's canal. Traditional glaucoma surgery has largely attempted to create alternative pathways for aqueous humour outflow by bypassing the TM and Schlemm's canal completely. Both trabeculectomy and conventional glaucoma drainage device surgery depend on creation of a fistula between the anterior chamber and subconjunctival space. Although conventional full thickness 'filtration' surgery provides very effective IOP reduction, allowing low target IOPs to be achieved, it carries the risk of over drainage and hypotony..$^{1.2}$ Also, as there is a communication between the subconjunctival space and anterior chamber, there is an on-going risk of intraocular infection, particularly in those with thin walled blebs. For these reasons, trabeculectomy and traditional tube surgery are typically reserved for patients with moderate to advanced glaucoma failing to obtain target IOP or progressing on maximal medical treatment. ${ }^{3}$

Despite the importance of the drainage angle in the pathogenesis of glaucoma, until recently the angle has been overlooked as a potential surgical target in adults. Angle procedures such as goniotomy and trabeculotomy have though been the preferred surgical option for children with primary congenital glaucoma (PCG). The reason for the different treatment approach is that there are distinct differences between POAG and PCG, with the latter the result of trabeculodygenesis; perhaps explaining the lack of efficacy of goniotomy in adults compared to children., ${ }^{4,5}$ The last decade, has however, seen renewed interest in angle surgery for POAG, with the introduction of several minimally invasive glaucoma surgery (MIGS) procedures specifically targeting the angle. Although these procedures typically have modest IOP reduction compared to trabeculectomy, they have the advantage of being conjunctiva preserving ab interno procedures with high safety profiles and rapid recovery compared to traditional glaucoma surgery. Their good safety profile may lower the threshold for surgery and provide a safer surgical option, more suitable for patients with mild to moderate glaucoma. While some MIGS devices target other anterior drainage pathways (such as the Xen ${ }^{\circledR}$ microstent, Allergan, CA, US), this review presents an overview of MIGS devices targeting the angle, as summarised in Table 1.

\section{Aqueous humour outflow}

The TM is a tiny triangle of connective tissue beams covered by TM cells located in the iridocorneal angle. It consists of three regions; (1) the uveal meshwork, which is the innermost portion of the TM, closest to the anterior chamber and extending from the iris root and ciliary body to the peripheral cornea, (2) the corneoscleral meshwork, which extends from the scleral spur to the anterior wall of the scleral sulcus, and (3) the outermost juxtacanalicular meshwork, which although being the thinnest part of the TM $(2-20 \mu \mathrm{m})$, contributes most to outflow resistance due to narrow intercellular spaces and dense extracellular matrix. Aqueous passes through the TM and inner wall of endothelial cells of Schlemm's canal to enter one of approximately 35 collector channels, before entering the aqueous veins and intrascleral and deep scleral venous drainage systems, and the episcleral veins where the pressure is approximately $8-10 \mathrm{mmHg}$. Outflow 
Figure 1: iStent (Glaukos)

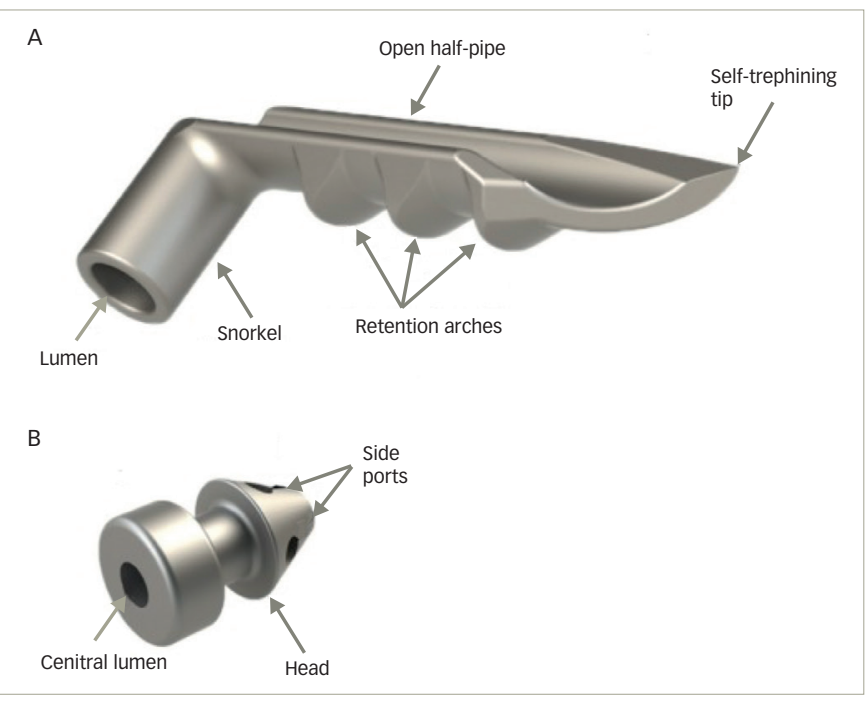

Reproduced with permission from Glaukos Corporation, CA, US.

is highly segmental meaning only a portion of the outflow pathway is active in fluid passage at any one time. Normal aqueous humour outflow occurs at a rate of $2.4 \pm 0.6 \mu \mathrm{l} / \mathrm{min}$ for a turnover of $1-1.5 \%$ of anterior chamber volume per minute. ${ }^{\circ}$

\section{Devices and outcomes}

\section{iStent and istent-inject}

The istent ${ }^{\circledR}$ Trabecular Micro-Bypass Stent (Glaukos Corporation, CA, US) was introduced in 2007 and approved by the FDA in 2012. It is a $0.3 \mathrm{~mm}$ wide, $1 \mathrm{~mm}$ long, heparin-coated, titanium, snorkel-like device which can be implanted through a $1.5 \mathrm{~mm}$ corneal incision (Figure 1). The device is implanted under gonioscopic guidance by pushing the tip of the stent through the TM into Schlemm's canal. This allows aqueous humour to drain from the anterior chamber into Schlemm's canal, bypassing two sites of resistance to aqueous humour outflow; the juxtacanalicular TM and the inner wall of endothelial cells of Schlemm's canal. However, successful IOP-lowering depends on the integrity of the distal outflow pathways. If the device is placed in a non-functioning section of schlemm's canal or if the collector channels or aqueous veins draining this section are compromised, the chances of success are likely reduced. istent is commonly combined with cataract surgery, so many of the studies examining the safety and efficacy of the device have compared combined cataract and istent surgery to cataract surgery alone.

A prospective multicentre, randomised clinical trial including 239 eyes compared the IOP-lowering effect of cataract surgery combined with istent to cataract surgery alone. The trial included patients with mild to moderate glaucoma with an un-medicated IOP between 22 and 36 $\mathrm{mmHg}$. After 2-years follow up, significantly more patients receiving istent had an IOP $\leq 21 \mathrm{mmHg}$ off medications (61\%), compared to those having cataract surgery alone $(50 \%, p=0.036)$. Patients receiving istent were also using significantly fewer medications at 1 year $(0.2 \pm 0.6)$ than the cataract surgery alone group $(0.4 \pm 0.7, p=0.011)$. However, there was no significant difference in medication use between drops at 2 years, and the mean IOP at 2 years was similar in both groups at approximately $17 \mathrm{mmHg}$. There was no difference between groups regarding postoperative complications. ${ }^{\text {? }}$

A recent meta-analysis of 32 articles published between 2000 to 2014, inclusive of 2,143 patients and eight randomised control trials, further
Table 1: Summary table of MIGS devices that target angle structures

\begin{tabular}{|l|l|}
\hline Device/technique & Drainage route/method of action \\
\hline iStent (Glaukos) & Via Schlemm's canal: bypass trabecular meshwork \\
\hline iStent Inject (Glaukos) & Via Schlemm's canal: bypass trabecular meshwork \\
\hline Trabectome (NeoMedix) & $\begin{array}{l}\text { Via Schlemm's canal: excision of trabecular } \\
\text { meshwork }\end{array}$ \\
\hline Cypass (Alcon) & Via suprachoroidal space \\
\hline Hydrus (Ivantis) & $\begin{array}{l}\text { Via Schlemm's canal: excision of trabecular } \\
\text { meshwork }\end{array}$ \\
\hline $\begin{array}{l}\text { AbIC: Ab Interno } \\
\text { Canaloplasty with iTrack } \\
\text { (Ellex) }\end{array}$ & $\begin{array}{l}\text { Via Schlemm's canal: dilatation of trabecular } \\
\text { meshwork }\end{array}$ \\
\hline \begin{tabular}{l} 
MIGS = minimally invasive glaucoma surgery. \\
\hline
\end{tabular}
\end{tabular}

examined the efficacy of a combined procedure with cataract surgery alone. ${ }^{8}$ The results showed a mean decrease in IOP of $9 \%$ from baseline following combined cataract surgery with one istent, compared to a $4 \%$ drop with cataract surgery alone.

Although istent does not have the same IOP reducing power compared to more invasive procedures, it has a good safety profile and may be considered for patients with mild to moderate disease, particularly those who also require cataract surgery. A second-generation istent (GTS400 iStent inject ${ }^{\oplus}$; Glaukos Corporation, CA, US) is now available, with two stents housed within the same injector, enabling two implants to be inserted with greater ease. This has potential advantages as there is some evidence that inserting two istents may offer superior IOP lowering compared to one istent alone. The istent meta-analysis found a $27 \%$ reduction in IOP with cataract surgery and two istents compared to a $9 \%$ reduction from baseline with cataract surgery and one stent. ${ }^{8}$ Initial results from a non-randomised, uncontrolled prospective case series of 20 patients, evaluating the efficacy and safety of istent inject with phacoemulsification were published recently. ${ }^{9}$ The study showed a $37 \%$ reduction in IOP $(9.74 \pm 3.14 \mathrm{mmHg}, \mathrm{p}<0.001)$ and a reduction in drops from $1.3 \pm 0.66$ to $0.75 \pm 0.79(p=0.017)$.

\section{Trabectome}

Trabectome ${ }^{\circledR}$ (NeoMedix Corp, CA, US) was the first of the modern devices for angle surgery, approved by the FDA in 2004. The procedure, which is also often combined with cataract surgery, involves creating a $1.6 \mathrm{~mm}$ clear corneal incision to perform electro-ablation of TM. Under direct visualisation through a surgical gonioscope, a bipolar electrode is used to deliver plasma-mediated ablation of TM, which reportedly minimises heat dissipation to surrounding tissues. Schlemm's canal is effectively de-roofed, therefore, similar to istent, Trabectome aims to remove resistance to aqueous outflow at the level of the TM and inner wall of Schlemm's canal. Trabectome is generally contraindicated for neovascular glaucomas, more than moderately active uveitis, angle dysgenesis and corneal oedema where the view of the angle is limited.

Being a more mature member of the MIGS family, there have been numerous studies assessing the efficacy of Trabectome. The largest study, carried out by the Trabectome Study Group, was a retrospective analysis of 1,127 eyes treated with either Trabectome alone or combined Trabectome and cataract surgery. ${ }^{10}$ Subjects varied significantly in age and diagnosis. Overall, there was a significant $39 \%$ reduction $(p<0.01)$ in mean pre-operative IOP $(23.8 \pm 7.7 \mathrm{mmHg}$ to $16.5 \pm 4.0 \mathrm{mmHg})$ at 24 -months follow-up. Trabectome alone achieved similar IOP reduction to combined 
Figure 2: Illustration of CyPass device insertion

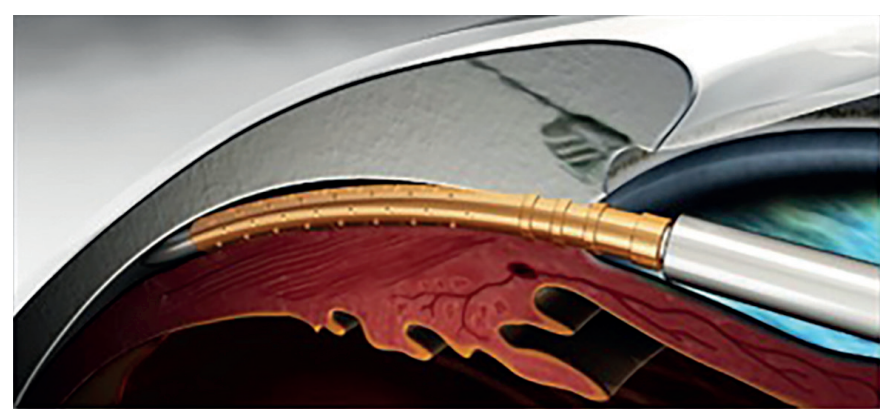

Reproduced with permission from Alcon, Texas, US.

Trabectome and cataract surgery. $75 \%$ of patients had 'intraoperative blood reflux' resulting in transient hyphaema and day-1 post-operative IOP spike (>10 mmHg above baseline) was noted in 65 of 1,127 cases. There were no reported cases of endophthalmitis, choroidal effusions or significant loss of visual acuity. Further analysis of the Trabectome Study Group data, alongside smaller-scale studies, suggests a typical IOP reduction with Trabectome of $18-40 \%$, with an average postoperative IOP of $15.3-17.4 \mathrm{mmHg}$, and a $40 \%$ reduction in medication use. ${ }^{11}$

\section{CyPass}

The CyPass $^{\circledast}$ (Alcon, Texas, US) is a minimally invasive ab interno device inserted between the sclera spur and ciliary body, taking advantage of the negative pressure gradient between the suprachoroidal space and the anterior chamber which facilitates drainage of aqueous into the suprachoroidal space. The device is $6.35 \mathrm{~mm}$ long, with an external diameter of $510 \mu \mathrm{m}$, and designed to be performed during cataract surgery. It received FDA approval in August 2016 (Figure 2).

The pivotal study evaluating Cypass was the 2-year COMPASS study, carried out by the CyPass Study Group, which evaluated the safety and efficacy of CyPass in patients also undergoing cataract surgery. ${ }^{12}$ Five-hundred and five participants were randomised into those having cataract surgery alone $(n=131)$ or those having combined cataract surgery with CyPass $(n=374)$. Subjects had a diagnosis of POAG with mean un-medicated IOP between 21-33 mmHg. The main outcome measures included the percentage of subjects achieving $\geq 20 \%$ unmedicated IOP lowering, mean IOP change and glaucoma medication use at 24 months. Overall, the study showed enhanced IOP-lowering with CyPass compared to cataract surgery alone. On average IOP was reduced by $7.4 \mathrm{mmHg}$ in the CyPass group compared to $5.4 \mathrm{mmHg}$ with cataract surgery alone $(p<0.001)$, with $85 \%$ of patients in the CyPass group not requiring glaucoma medications, compared to $59 \%$ of controls. Rates of adverse events were similar between groups, with CyPass exhibiting a good safety profile.

\section{Hydrus Microstent}

The Hydrus Microstent'M (Ivantis, CA, US) is a trabecular bypass device that provides a 'scaffold' for Schlemm's canal. Made from nitinol, an alloy of nickel and titanium, it measures $8 \mathrm{~mm}$ in length and is crescenteric in shape, allowing it to cannulate approximately three clock-hours of Schlemm's canal. This coverage allows increased coverage of collector channels which is likely to maximise the chances of finding an intact distal outflow pathway. Ex vivo studies in animal and cadaveric eyes have shown increased outflow when compared to istent. ${ }^{13}$

The HYDRUS II study was a 2-year prospective, single-masked multicentre trial comparing the Hydrus Microstent combined with cataract surgery to cataract surgery alone. ${ }^{14}$ Results obtained from 78 subjects showed $80 \%$ of patients achieved a $20 \%$ reduction in IOP with Hydrus plus cataract surgery compared to $46 \%$ with cataract surgery alone. Average IOP was also lower in the Hydrus group $(16.9 \pm 3.3 \mathrm{mmHg}$ compared to $19.2 \pm 4.7 \mathrm{mmHg}, \mathrm{p}=0.009$ ). The proportion of patients who were medication-free at 24-months was higher with Hydrus by a factor of almost 2:1 (72.9\% versus $37.8 \%, p=0.0008)$, and no serious adverse events were encountered in either group.

\section{Kahook Dual Blade}

The Kahook Dual Blade (New World Medical, California, US) is a novel single-use ab interno device that has a pointed tip to pierce the TM, a ramp to lift the tissue above the normal plane of the TM, and two parallel blades to incise the TM and inner wall of Schlemm's canal, without residual TM or damage to surrounding structure. In cadaver eyes, TM excision with the dual blade was more complete than with the Trabectome, ${ }^{15}$ however, results from clinical studies are awaited.

\section{Canaloplasty (ab externo) and AbIC (ab interno)}

Traditional canaloplasty requires a partial conjunctival peritomy and scleral flap formation to gain success to Schlemm's canal. Through this access, a small catheter is inserted and viscoeleastic dilatation of schlemm's canal is achieved throughout its full length. However, in order to maintain adequate dilation a tight intraluminal suture is required. This procedure requires conjunctival sacrifice, a sclerostomy, and the risk of Schlemm's canal recollapse following the procedure. Ab interno canaloplasty is a refined version of the ab externo approach. Through a $1.8 \mathrm{~mm}$ clear corneal incision and under gonioscopic vision, access to schlemms canal is gained using through a small goniotomy. A microcatheter is then advanced the full length of Schlemm's canal, under transscleral visualisation provided from the illuminated catheter tip. Once fully accessed Schlemm's canal is viscodilated, which not only dilates the canal but also the collector channels. ${ }^{16}$ Whilst the manufacturers highlight the ab interno approach is as effective as traditional canaloplasty, at present, there are few published studies available.

\section{The future of angle surgery}

The angle has had somewhat of resurgence as a target for glaucoma surgery, and now offers itself as a viable site for safe, minimally invasive ab interno techniques. While trabeculectomy and conventional tube surgery offer a superior pressure-lowering profile, they also carry greater risk of complications, meaning they tend to be reserved for patients who have exhausted other treatment options or have advanced disease. MIGS devices can be offered earlier in the course of disease, reducing the burden of medications, and perhaps delaying or avoiding the need for conventional glaucoma surgery. The renewed interest in minimally invasive surgical techniques targeting the angle has become an exciting and dynamic area of surgical progress in glaucoma. $\square$ management of glaucoma, Optom Vis SCi, 2011;88:E39-47. Gedde S, Herndon IW, Brandt JD, et al. Postoperative complications in the tube versus tra, complications in the tube versus trabeculectomy (IVT) 2012:153:804-14. 2012,153:804-14.

Kirwan JF, Lockwood AJ, Shah P, et al, Trabeculecomty in the 21st century: a multicenter analysis, Ophthalmology,
4. Yan Yu Chan J, Bonnie NK Choy, Alex LK Ng Jennifer WH Shum, Review on management of primary congenital glaucoma, J Curr Glaucoma Pract, 2015:9:92-9.

Mendicino ME, Lynch MG, Drack A, et al., Long-term surgical and visual outcomes in primary congenital glaucoma: 360 degrees trabeculotomy versus goniotomy, J AAPOS, 2000;4:205-10.
6. Abu-Hassan DW, Acott TS, Kelley MJ, The trabecular meshwork: a basic review of form and function J Ocul Biol, 2014;2.

Craven ER, Katz $L$, Wells JM, Giamporcaro JE, istent Study Group; Cataract surgery with trabecular micro-bypass stent implantation in patients with mild-to-moderate open-angle glaucoma and cataract: Two-year follow-up, J Cataract Refract glaucoma and cataract:
Surg, 2012;38:1339-45. 
8. Malvankar-Mehta MS, Iordanous Y, Chen YN, et al., istent with phacoemulsification versus phacoemulsification alone for phacoes patients with glaucoma and 2015:10:e0131770.

CNE, 2015, Glaukos istent inject ${ }^{\oplus}$ trabecular micro-bypass implantation associated with cataract surgery in patients with coexisting cataract and open-angle glaucoma or ocular hypertension: long-term study, J Ophthalmol, 2016;1056573.Epub.

10. Minckler D, Mosaed S, Dustin L, et al. Trabectome (trabeculectomy-internal approach): additional experience and extended follow-up, Trans Am Ophthalmol Soc, 2008;106:149-60

11. Brandão $L M$, Grieshaber $M C$, Update on Minimally Invasive Glaucoma Surgery (MIGS) and New Implants, I Ophthalmol, Glaucoma Surgery

12. Vold $\mathrm{S}$, Ahmed II, Craven ER, et al., Two-year COMPASS trial results: supraciliary microstenting with phacoemulsification in patients with open-angle glaucoma and cataracts, Ophthalmology, 2016;123:2103-12.

13. Hays $\mathrm{CL}$, Gulati $\mathrm{V}$, Fan $\mathrm{S}$, et al., Improvement in outflow facility by two novel microinvasive glaucoma surgery implants,
Invest Ophthalmol Vis Sci, 2014:55:1893-900.

14. Pfeiffer N, Garcia-Feijoo J, Martinez-de-la-Casa JM, et al., A randomized triat of a schlemm's cant microstent with phacoemulsification for reducing intraocular pressure in openangle glaucoma, Ophthalmology, 2015;122:1283-93.

15. Seibold LK, Soohoo JR, Ammar DA, Kahook MY, Preclinical investigation of ab interno trabeculectomy using a novel dualblade device, Am J Ophthalmol, 2013;155:524-9.

16. Khaimi MA, Canaloplasty; a minimally invasive and maximally effective glaucoma treatment, Journal of Ophthalmology, 2015; Article ID 485065 